\title{
Degradación por sobre-pastoreo de los pastos nativos en la quebrada de Quillcayhuanca
}

Degradation by over-pastoring of the native pastures in the ravine the Quillcayhuanca

Hernán Vega Mejia ${ }^{1}$

\section{RESUMEN}

El objetivo de la investigación es identificar el nivel de degradación de los pastos nativos por el excesivo pastoreo, presentando un franco proceso de degradación de los pastos de mejor calidad de la quebrada de Quillcayhuanca, principalmente por especies exóticas como el ganado ovino, vacuno y equinos. Para identificar los estratos y las unidades primarias de muestreo-UPM se ha construido un marco de muestreo tomando como criterio de estratificación la conformación de los sitios de pastoreo propuesto por el estudio ejecutado por el Parque Nacional Huascarán, Instituto Nacional de Recursos Naturales. Para el censo de la cobertura vegetal se ha utilizado el anillo censador de $2 \mathrm{~cm}$ de diámetro, con la finalidad de obtener e identificar las especies por cada uno de los 19 sitios, se tomaron las muestras con el anillo censador de acuerdo al número estimado de transeptos que cubren al área de cada sitio. Así también se obtuvo la información histórica y actual de la población de ganado existente en la quebrada, obteniendo información desde el año 1975 al año 2008. Con la información generada e histórica y el análisis estadístico correspondiente, presentan como resultado un alto grado de correlación con un promedio de aproximadamente de 0.89 entre la soportabilidad de los pastos nativos y el incremento de la población ganadera exótica, resultados que permiten plantear una serie de recomendaciones teniendo en cuenta la recuperación natural de la pradera nativa sobre todo el equilibrio del ecosistema.

Palabras clave: degradación; sobrepastoreo; pastos nativos.

1 Universidad Nacional «Santiago Antúnez de Mayolo». Huaraz, Perú. 


\section{ABSTRACT}

The research identifies the level of native grasses degradation due to excessive grazing, presenting a frank degradation process of the best quality grazing pastures of Quillcayhuanca, mainly by exotic species such as sheep, cattle and horses.

To identify the strata and the primary units of sampling-UPM, a sampling frame has been constructed, taking as a stratification criterion the conformation of the grazing sites proposed by the study carried out by the Huascarán National Park (2000).

For the census of plant cover, the $2 \mathrm{~cm}$ diameter census ring was used to obtain and identify the species for each of the 19 sites. The samples were taken with the census ring according to the estimated number of species Transepts that cover the area of each site. The historical and current information on the cattle population in the creek was also obtained, obtaining information from 1975 to 2008.

With the information generated and historical and the corresponding statistical analysis, they present a high degree of correlation with an average of approximately 0.89 between the sustainability of the native pastures and the increase of the exotic cattle population, results that allow to raise a series of Recommendations taking into account the natural recovery of the native prairie over all ecosystem balance.

Keywords: degradation; overgrazing; native grasses.

\section{ICHIKLLACHAW}

Kay musyapakuyqa riqitsimantsik imanawmi allaapa mitsikuywan llapan hirkachaw pastukuna, qurakunata ushakuykanqantam, kay alli pastukunaqa Qillqaywanka raqrachawmi ushakaakuykan, tsayqa ushakan uushakunapa, kawallukunapa, ashnukunapa, waakakunapa mikuyninkunawanmi. Hinaman UPMnishqanta riqinapaqqa muyistriyu nishqanwanmi pallaritsikashqa, tsaypaq may tsaychawyan mitsiyanqantam wanakashqa, hinaman kaypaqqa Parki Nasiyunal Waskarantawan Institutu Nasiyunal Rikursus Naturalis nishqantam wanakashqa. Atska kaynin hachakuna musyanapaqqa siwi sinsadur 2cmpa diyamitruntam wanakashqa. Tsaynawpa 19 kutakunachaw llapan raqrakunachaw llapan ashmakuna kanqanta riqinapaq. Hinaman unaywan kanan patsachawwan llapan ashmakuna tsay raqrachaw kanqan willakuytam hurqaqashqa. Kay musyapakuyqa pallarikashqa 1975piq 2008 watakama kanqantam pallarikashqa. Tsay llapanwanmi huk hatun kurilasiyun nishqan yarqun, kayqa 0.89manmi chan, kikin pastukuna kakunqanwan mikuq ashmakuna kanqanwanpis. Tsayllapan musyayninwanmi alli willapayninta wanan, tsaynawpa ñawpa ñawpa pastukuna kanqantanawllaman kutinanpaq, tsaynawpa raqrakuna, hirkakuna allilla kawayaananpaq.

Pushaq shimikuna: pastukunapa ushakaynin; lluta mitsikuynin; ñawpa pastukunapa qurakunapa kawaynin. 


\section{INTRODUCCIÓN}

La estructura y funcionamiento de los ecosistemas como los que presenta la quebrada de Quillcayhuanca, son consecuencia de cambios progresivos que se producen al ser sometidos a modificaciones bióticas, resultado del uso indiscriminado a través de los años.

Los cambios producidos por el sobre-pastoreo, demuestran que los componentes del ecosistema, bajo condiciones naturales, son incapaces de sustituir la fitocenosis (Flores y Malpartida, 1987),motivo por el cual la investigación tiene el propósito de contribuir a la sostenibilidad de los ecosistemas generando información y nuevos conocimientos de los impactos negativos que soportan los pastos naturales a nivel de la quebrada de Quillcayhuanca y, plantear alternativas de remediación y sostenimiento del ecosistema.

La problemática de conservación y pérdida de las pasturas no es reciente, sino que se remonta a la época de la conquista, en que se introdujo el ganado ovino, vacuno y equino. El primer impacto negativo fue el despoblamiento total de la ganadería nativa, constituido por alpacas, llamas y vicuñas. La ganadería establecida se propagó y difundió, sin ningún control, produciéndose en primera instancia la degradación y degeneración del ganado introducido denominado «ganado criollo», que se caracteriza por la alta rusticidad adquirida; pero, en cambio, sus niveles de productividad son bajos, lo cual lo convierte en depredador del recurso vegetal. Se hace necesario disponer de información que nos determine el grado de degradación generado por el sobrepastoreo en la quebrada de Quillcayhuanca para poder determinar el manejo más eficiente y eficaz de los pastos nativos. Entonces, el objetivo es identificar el índice de vigor del pastizal o índice de soportabilidad (Salvador, 2002), método usado en la presente investigación para poder identificar la degradación que cada vez se va incrementando por el excesivo pastoreo, complementado este impacto ambiental con un mínimo o ningún manejo técnico de los pastizales en el ámbito de la quebrada de Quillcayhuanca, la que cuenta con una superficie total de 1,725.77 hectáreas dedicadas al pastoreo indiscriminado.

Se presenta los procedimientos y resultados del análisis de la población pecuaria, la composición florística obtenida en varios periodos que van del año de 1975 al 2000, y generado para proyectarlo al año 2008 bajo los procedimientos metodológicos aplicados en la investigación. Con toda la información procesada se obtuvo el índice de correlación entre la población ganadera asentada en la quebrada y los pastos naturales, así como el grado de degradación del pastizal, el cual ha sido determinado basándonos en la capacidad de carga, de lo cual se obtiene la oferta forrajera en base verde.

Seguidamente, el artículo presenta los métodos y procedimientos que condujeron a los resultados cuantitativos y de áreas, información básica para la obtención del grado de degradación de los pastos nativos en la quebrada de Quillcayhuanca. 


\section{MATERIALES Y MÉTODOS}

La metodología en el este trabajo de investigación es del tipo no experimental longitudinal, en la que se mide en varias ocasiones las variables involucradas como el número de cabezas de ganado por especie y la composición florística de la quebrada de Quillcayhuanca. De los archivos históricos del Parque Nacional Huascarán, se obtuvo la información de la población ganadera desde al año 1975 al 2002, datos generados por enumeración completa, mientras que los datos de la población de ganado para los años 2003 al 2008 se estimaron mediante modelo de regresión. Para la identificación y cuantificación del componente florístico de la quebrada de Quillcayhuanca, se levantó información haciendo uso del muestreo aleatorio estratificado. Para su ejecución, el territorio total fue dividido en 19 sectores o «sitios». En cada sector, para la obtención de las muestras, se empleó el método de «transacción al paso», que consiste en determinar la estructura de la vegetación en porcentaje, haciendo uso del anillo censador de $2 \mathrm{~cm}^{2}$ de área; cada transepto contiene información referente a lugar, sitio, fecha, porcentaje de pendiente, orientación, vegetación predominante, cobertura estimada y condición estimada. Con la información obtenida y generada se procedió a evaluar y comparar la tendencia que presenta el índice de soportabilidad del pastizal comparándolo con el obtenido por el Parque Nacional Huascarán el año 2000.

\section{RESULTADOS}

La quebrada de Quillcayhuanca se encuentra ubicada a 11 kilómetros al este de los distritos de Huaraz e Independencia; ocupa una extensión de 1725,77 Ha., que representa el 100\% del área total de la investigación.

La altitud de la quebrada va desde los 3800 hasta los 4200 msnm, a la zona de vida denominada bosque húmedo montano tropical (bh-MT) o sub-Páramo Tropical. Luego, sigue el fondo de la quebrada cuyo nivel alcanza aproximadamente los $4200 \mathrm{msnm}$, que corresponde a la zona de vida denominada páramo muy húmedo-Subalpino Tropical (pmh-Sa'T) según el Mapa Ecológico del Perú, Instituto Nacional de Recursos Naturales (1995).

Con la información de campo se obtuvo el inventario de la composición florística y la estimación de la presencia de cada especie en los 19 «sitios» de pastoreo.

La comunidad vegetal dominante en la quebrada Quillcayhuanca se encuentra caracterizada por la presencia de especies vegetales de las familias Gramínea, Ciperácea e, Juncaceae, Rosáceae, Leguminosae y Compositae. Estas se diferencian por su composición porcentual entre las especies de estrato alto y estrato bajo, según los resultados obtenidos de los 19 «sitios». La determinación de las condiciones del pastizal, se han calculado sobre la base de los índices de especies decrecientes y medidas de vigor (Flores, 2005), considerando niveles entre pobre y muy pobre la condición de los pastos naturales de la quebrada de Quillcayhuanca. 
Tabla 1. Composición florística por sitio y condición del pastizal por especie

\begin{tabular}{|c|c|c|c|c|c|c|}
\hline \multirow[t]{2}{*}{ Sitio } & \multirow[t]{2}{*}{ Superficie } & \multirow[t]{2}{*}{ Especie dominante } & \multicolumn{2}{|c|}{$\begin{array}{c}\text { Produccion de Biomasa } \\
\text { Kg.Mv./Ha. }\end{array}$} & \multicolumn{2}{|c|}{ Condicion de pastizal } \\
\hline & & & 2000 & 2008 & Ovinos & vacunos \\
\hline 1 & 84.37 & Muhlenbergia ligulares - Alchemilla & 600 & 641 & Pobre & Muy pobre \\
\hline 2 & 160.42 & Agrostis breviculmis -Alchemilla pinnata & 720 & 703 & Regular & Muy pobre \\
\hline 3 & 77.08 & Trifolium amabile - Alchemilla pinnata & 520 & 511 & Regular & Muy pobre \\
\hline 4 & 35.16 & Alchemilla pinnata - Bromus lanatus & 1,120 & 1,085 & Pobre & Muy pobre \\
\hline 5 & 177.34 & Alchemilla pinnata - Trifolium amabile & 800 & 788 & Regular & Muy pobre \\
\hline 6 & 44.79 & Trifolium amabile - Alchemilla pinnata & 1,000 & 962 & Regular & Muy pobre \\
\hline 7 & 65.62 & Alchemilla pinnata - Agrostis breviculmis & 1,120 & 1,031 & Pobre & Pobre \\
\hline 8 & 20.05 & Pedregosidad & 880 & 890 & Muy pobre & Muy pobre \\
\hline 9 & 36.2 & Alchemilla pinnata - Bromus lanatus & 1,200 & 1,175 & Pobre & Muy pobre \\
\hline 10 & 53.65 & Alchemilla pinnata - Agrostis breviculmis & 1,000 & 957 & Pobre & Muy pobre \\
\hline 11 & 40.1 & Agrostis breviculmis - Trifolium amabile & 480 & 469 & Pobre & Muy pobre \\
\hline 12 & 35.42 & Stipa brachiphylla & 1,600 & 1,487 & Regular & Pobre \\
\hline 13 & 84.9 & Alchemilla pinnata - Agrostis breviculmis & 1,400 & 1,397 & Pobre & Muy pobre \\
\hline 14 & 148.7 & Alchemilla pinnata - Festuca dolichophylla & 600 & 591 & Regular & Muy pobre \\
\hline 15 & 23.7 & Plantago rigida - Agrostis rigescens & 760 & 746 & Regular & Muy pobre \\
\hline 16 & 31.51 & Carees s.f. - Alemilla pinnata & 1,600 & 1,587 & Pobre & Muy pobre \\
\hline 17 & 90.88 & Luzula peruviana - Stipa brachiphylla & 720 & 729 & Pobre & Muy pobre \\
\hline 18 & 462.76 & Muhlenbergia fastigiata - Stipa brachiphylla & 4,800 & 4,832 & Pobre & Muy pobre \\
\hline 19 & 53.12 & Dissanthelium peruviana-Alchemilla pinnata & 1,520 & 1,537 & Pobre & Muy pobre \\
\hline Total & 1725.77 & & 22,440 & 22,118 & & \\
\hline
\end{tabular}

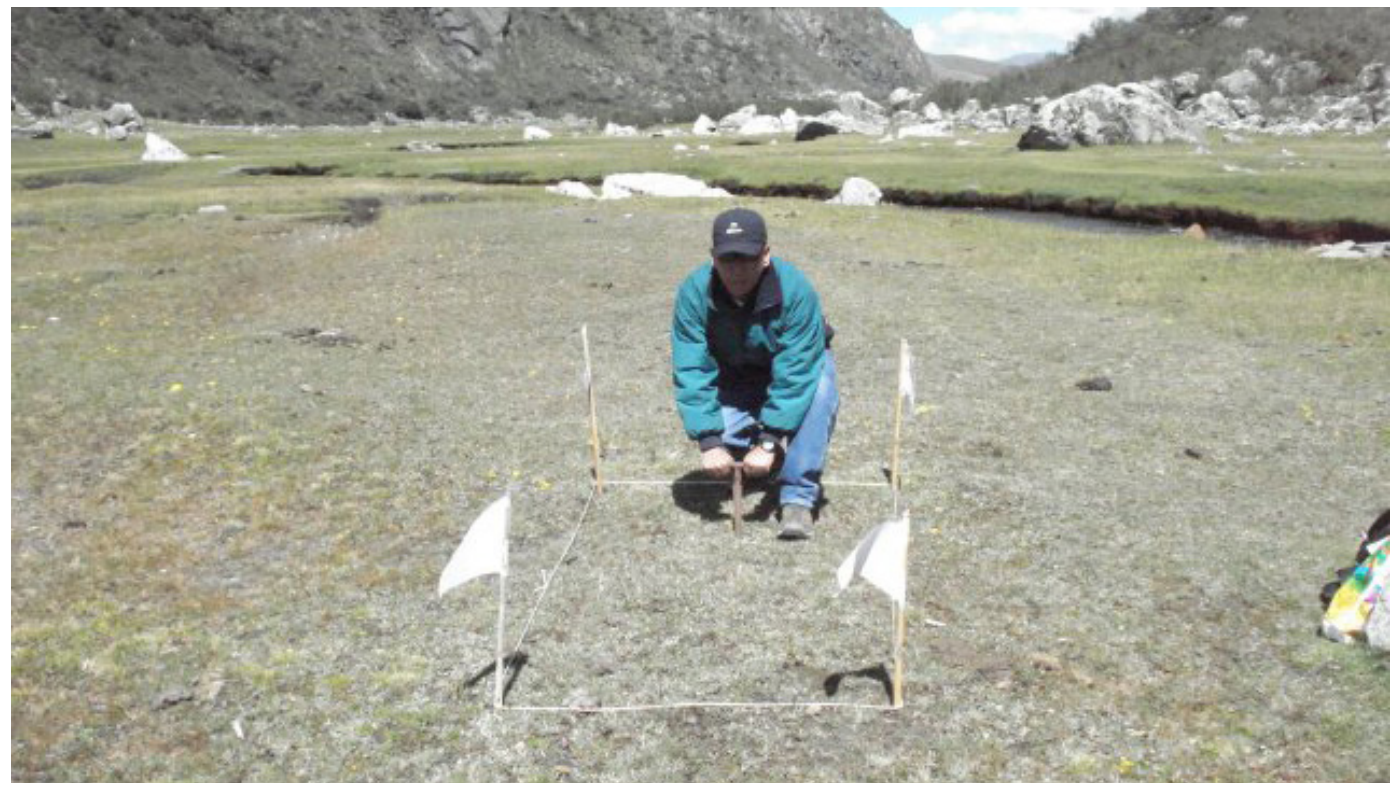

Figura 1. Transepto seleccionado y uso del anillo censador 
Tabla 2. Sitios, carga animal y soportabilidad del pastizal

\begin{tabular}{|c|c|c|c|c|}
\hline \multirow{2}{*}{ Sitio } & \multicolumn{4}{|c|}{ Soportabilidad del pastizal } \\
\hline & \multicolumn{4}{|c|}{ Ovinos* Vacunos* Ovinos** Vacunos** } \\
\hline 1 & 1.4 & 0.12 & 118 & 10 \\
\hline 2 & 0.5 & 0.06 & 74 & 10 \\
\hline 3 & 0.4 & 0.06 & 31 & 5 \\
\hline 4 & 0.5 & 0.06 & 18 & 2 \\
\hline 5 & 0.4 & 0.06 & 71 & 11 \\
\hline 6 & 0.4 & 0.06 & 18 & 3 \\
\hline 7 & 1.4 & 0.06 & 92 & 4 \\
\hline 8 & 0.4 & 0.06 & 8 & 1 \\
\hline 9 & 1.4 & 0.06 & 51 & 2 \\
\hline 10 & 1.4 & 0.06 & 75 & 3 \\
\hline 11 & 1.4 & 0.06 & 56 & 2 \\
\hline 12 & 0.4 & 0.06 & 14 & 2 \\
\hline 13 & 1.4 & 0.06 & 119 & 5 \\
\hline 14 & 0.4 & 0.06 & 59 & 9 \\
\hline 15 & 0.3 & 0.06 & 6 & 1 \\
\hline 16 & 1.3 & 0.06 & 41 & 2 \\
\hline 17 & 0.4 & 0.06 & 36 & 5 \\
\hline 18 & 1.4 & 0.12 & 648 & 56 \\
\hline 19 & 1.4 & 0.12 & 74 & 6 \\
\hline Total & & & 1609 & 139 \\
\hline Prom & 0.87 & 0.07 & & \\
\hline
\end{tabular}
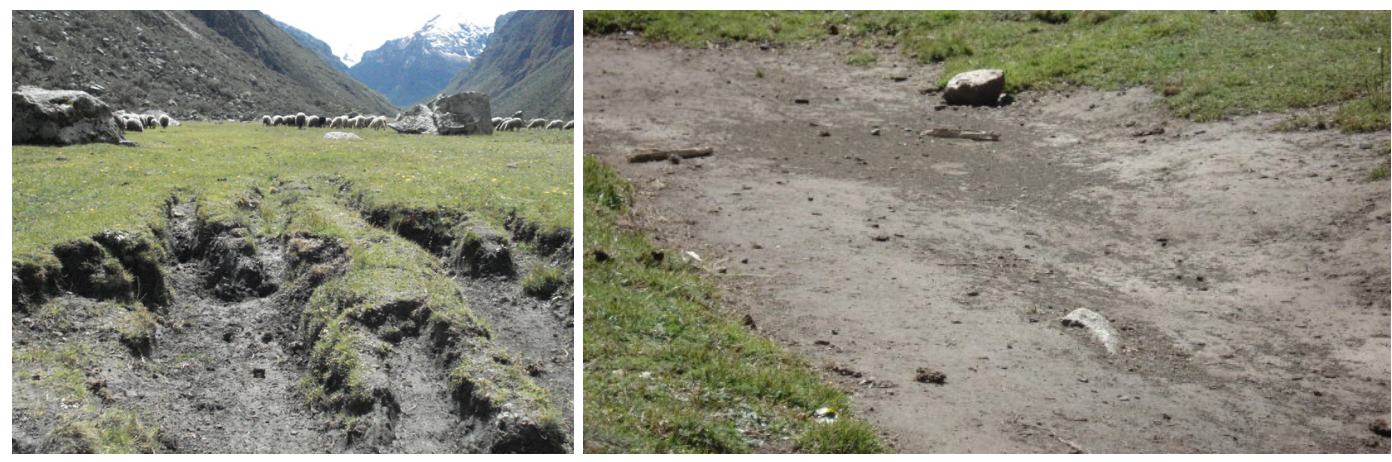

Figura 2. Área pisoteada por el ganado ovino Figura 3. Área desnuda en aumento, sin capacidad de regeneración

Considerando las dos especies de mayor importancia en cada sitio, los resultados nos indican las condiciones del pastizal y nos sugieren un pastoreo excluyente; se calcula la soportabilidad de 1609 y 139 unidades de ovinos y vacunos, respectivamente. 
Degradación por sobre-pastoreo de los pastos nativos en la quebrada de Quillcayhuanca||

Tabla 3. Población ganadera por especies $1975-2008$

\begin{tabular}{lrrrrrrrrrr}
\hline CANTIDAD DE & \multicolumn{10}{c}{ AÑOS } \\
\hline \multicolumn{1}{c}{ GANADO } & $\mathbf{1 9 7 5}$ & $\mathbf{1 9 7 9}$ & $\mathbf{1 9 9 9}$ & $\mathbf{2 0 0 0}$ & $\mathbf{2 0 0 1}$ & $\mathbf{2 0 0 2}$ & $\mathbf{2 0 0 5}$ & $\mathbf{2 0 0 6}$ & $\mathbf{2 0 0 7}$ & $\mathbf{2 0 0 8}$ \\
\hline VACUNO & 472 & 394 & 1413 & 1008 & 1320 & 1320 & 1369 & 1713 & 1831 & 1948 \\
OVINO & 78 & 6 & 264 & 178 & 251 & 251 & 276 & 351 & 380 & 408 \\
EQUINO & 57 & 53 & 318 & 211 & 297 & 297 & 312 & 407 & 439 & 472 \\
\hline TOTAL & 607 & 453 & 1995 & 1397 & 1868 & 1868 & 1957 & 2471 & 2650 & 2828 \\
\hline
\end{tabular}
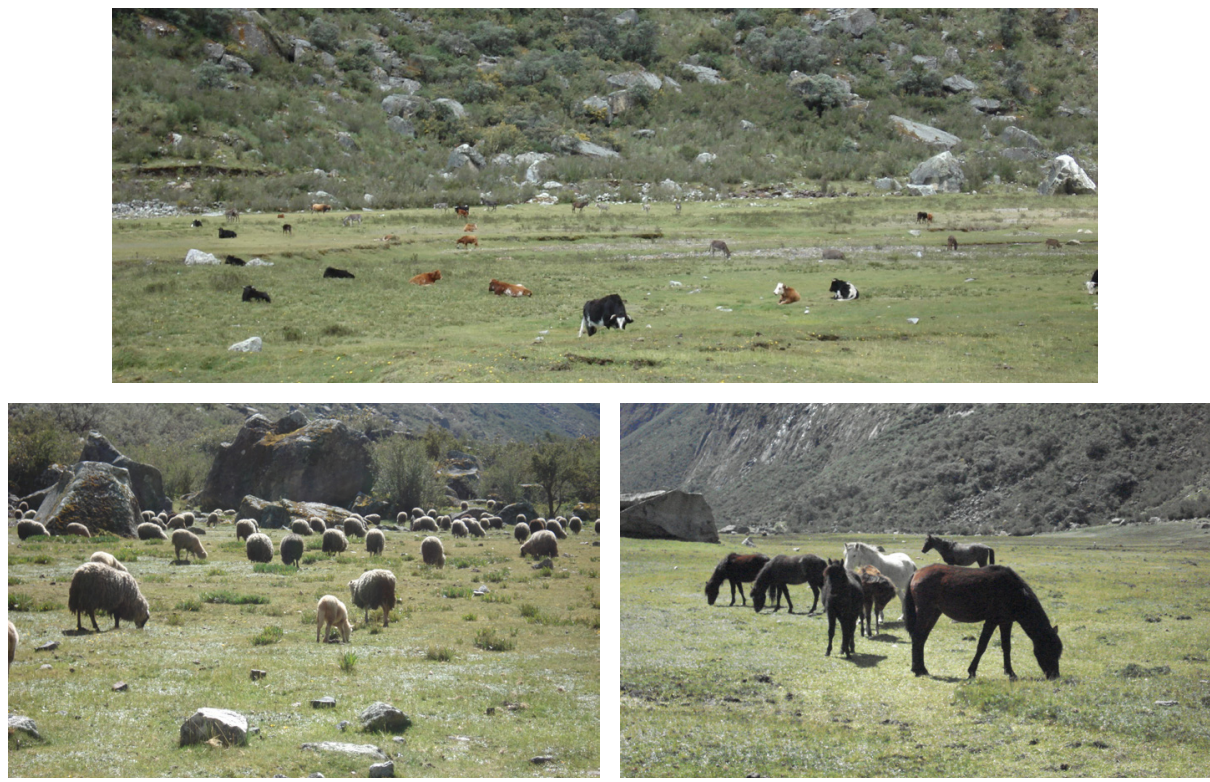

Figura 4. 5. 6. Ganado ovino, vacuno y equino asentado en la quebrada de Quillcayhuanca

Los mapas agrostológicos generados basándonos en las imágenes de satélite y ratificadas en campo se presentan a una escala de 1:50 000, con lo cual se muestra las asociaciones agrostológicas, áreas de ríos y lagunas. Las asociaciones se han definido representando la condición del pastizal por «sitio». El mapa agrostológico nos muestra que los pastizales se encuentran en las peores condiciones cualitativas, definidas para los pastizales, mostrando objetivamente que los pastos nativos de la quebrada de Quillcayhuanca se encuentran en un proceso de degradación. Comparando el mapa agrostológico elaborado por el Parque Huascarán en el Instituto Nacional de Recursos Naturales (2000), se observa que la condición del pastizal se encontraba en mejor estado, ya que se muestra una superficie que representa aproximadamente el $45 \%$ con una superficie con pastos considerando su condición como regular. Actualmente los pastos nativos se encuentran en un estado de regular, malo y muy malo para la soportabilidad del ganado ovino, lo cual ahonda el problema para soportar al ganado vacuno que presenta una calidad de malo a muy malo. 


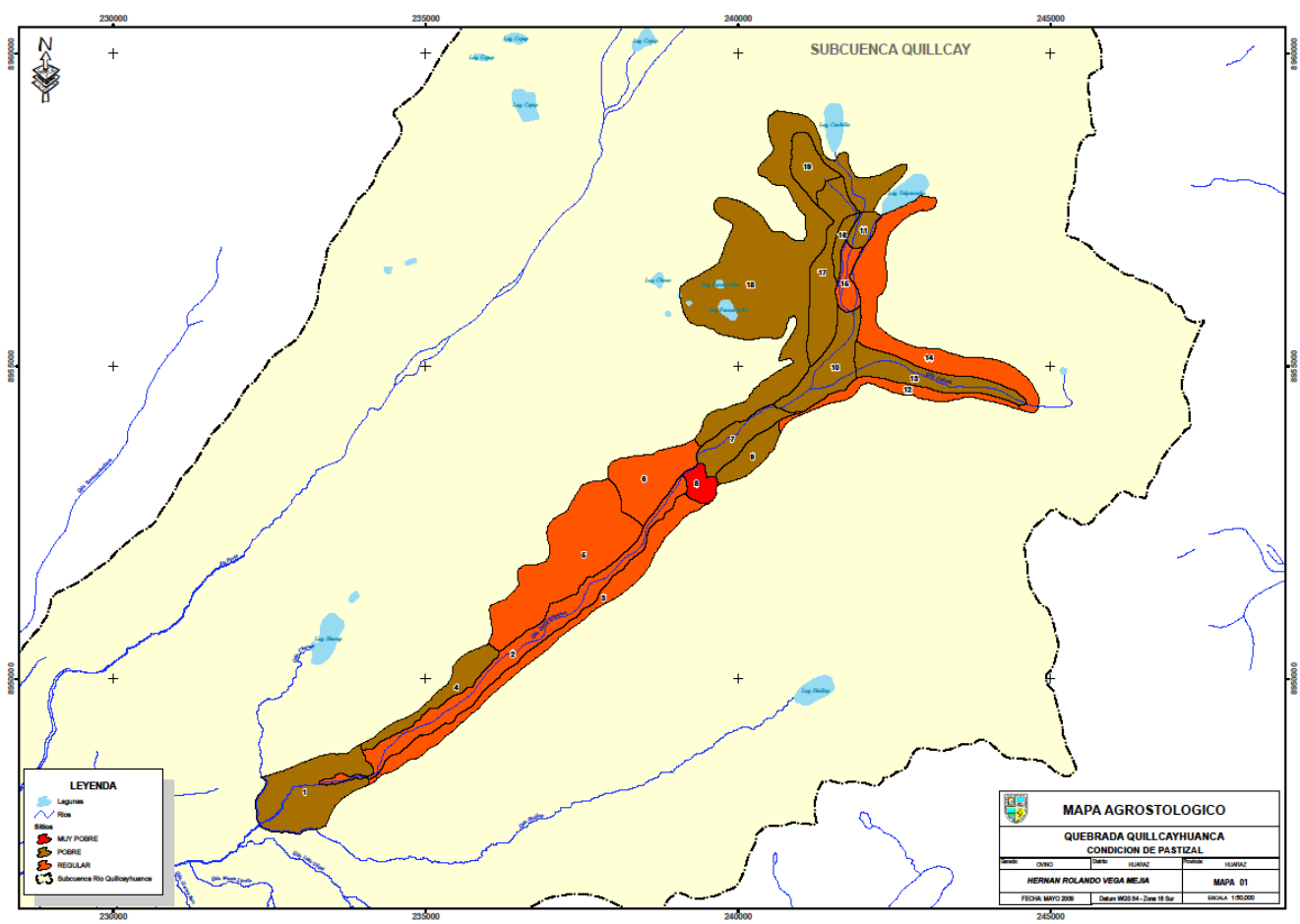

Figura 7. Mapa agrostológico. Quebrada Quillcayhuanca: condición del pastizal para ovinos

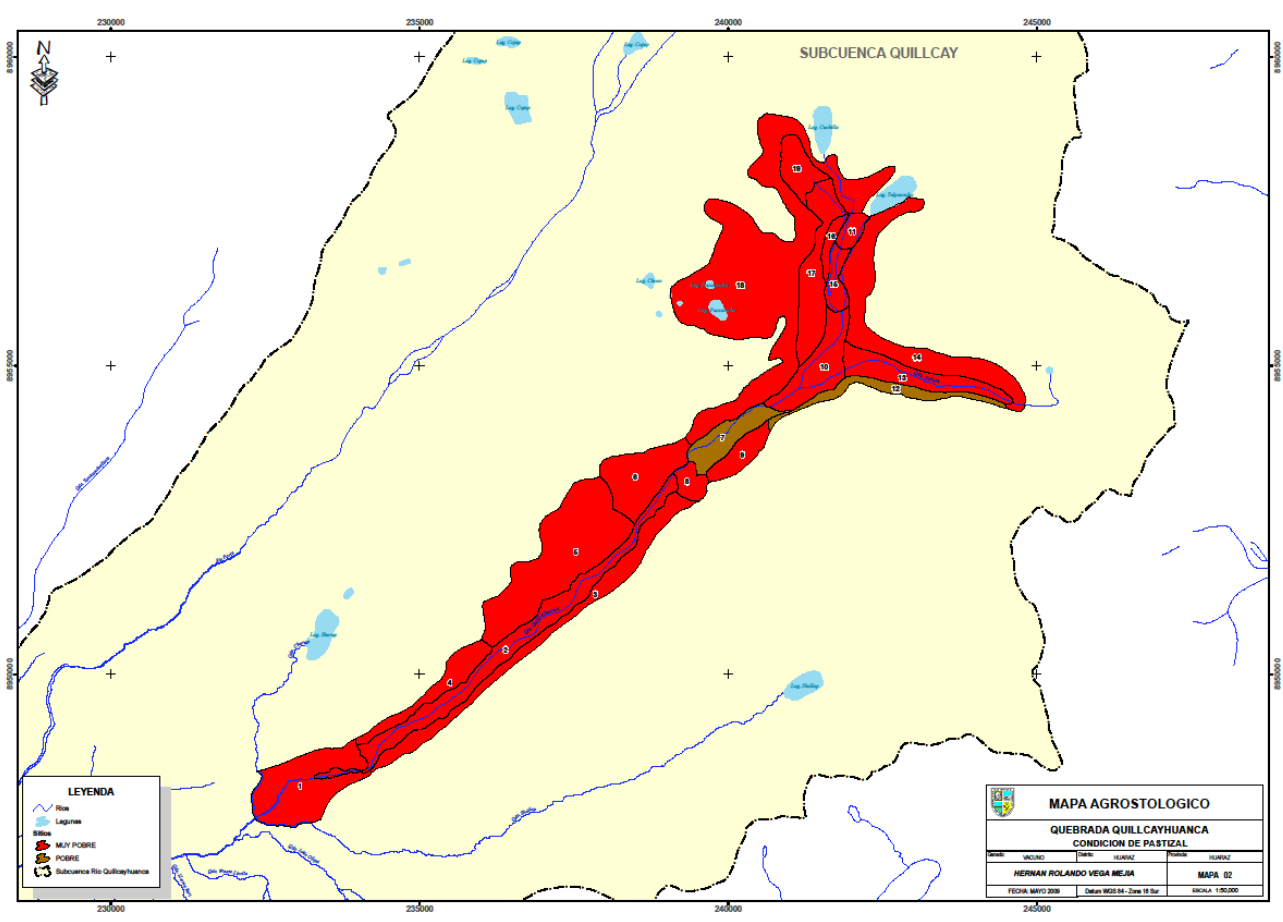

Figura 8. Mapa agrostológico. Quebrada Quillcayhuanca: condición del pastizal vacuno 


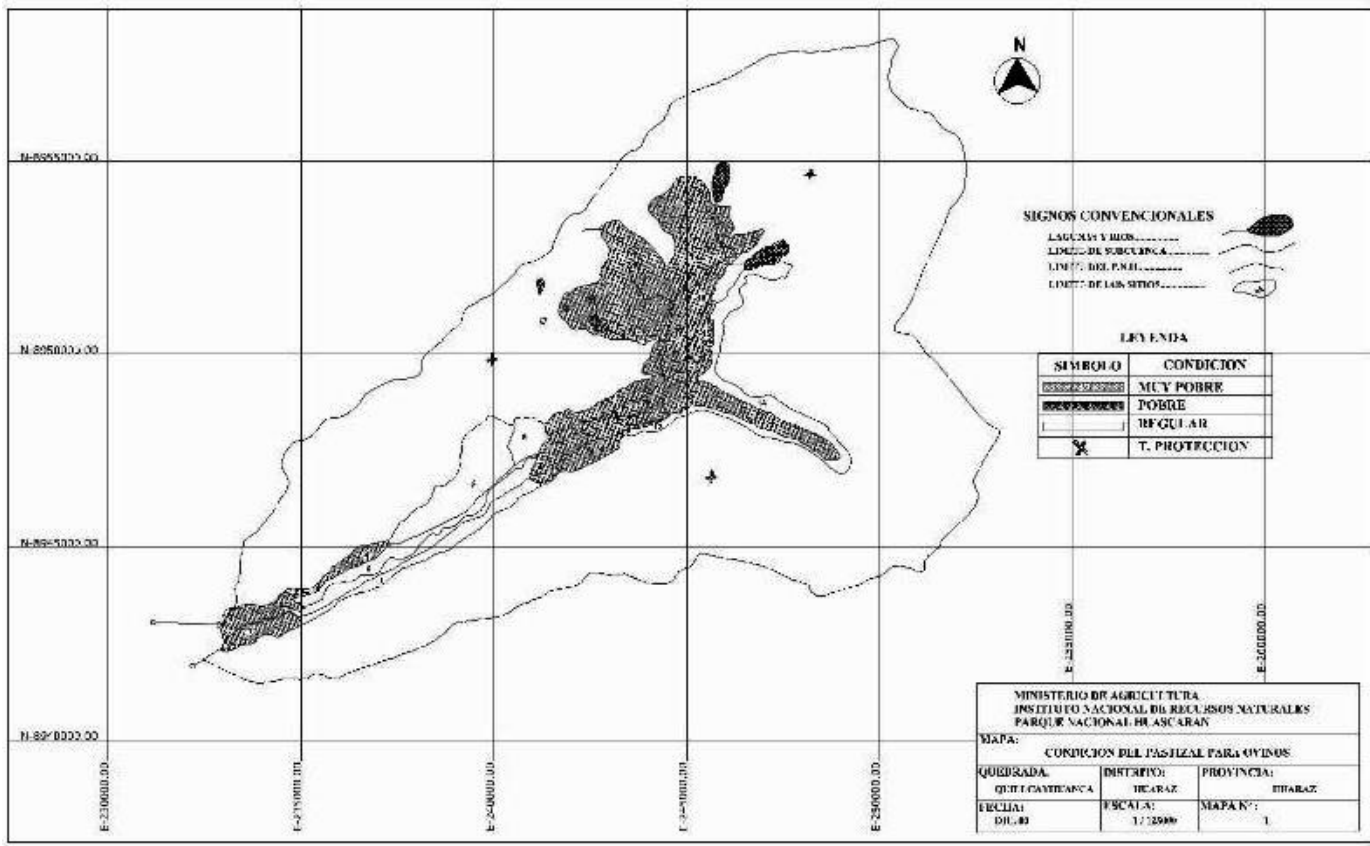

Figura 9. Mapa agrostológico. Quebrada Quillcayhuanca - PNH. 2000

La información de la población ganadera desde los años 1975 al 2005, la de soportabilidad de los pastos del año 2000 y la generada los años 2007 - 2008, así como la evolución de la población desde el año 1975, sirvieron para generar y obtener el grado de correlación entre la soportabilidad de los pastos y el incremento de la población ganadera.

\begin{tabular}{llrrrr}
\multicolumn{5}{c}{ Correlations } \\
\hline \multirow{5}{*}{ Pearson Correlation } & SOPVAC & CANTVAC & CANTOVI & CANTCAB \\
& SOFVAC & 1.000 & -.837 & -.890 & .939 \\
& CANTVAC & -.837 & 1.000 & .994 & .952 \\
& CANTOVI & -.890 & .994 & 1.000 & .973 \\
& CANTCAB & -.939 & .952 & .973 & 1.000 \\
Sig. (1-tailed) & SOPVAC & .000 & .000 & .000 & .000 \\
& CANTVAC & .000 & .000 & .000 & .000 \\
& CANTOVI & .000 & .000 & .000 & .000 \\
& CANTCAB & 34 & 34 & 34 & 34 \\
& SOPVAC & 34 & 34 & 34 & 34 \\
& CANTVAC & 34 & 34 & 34 & 34 \\
& CANTOVI & 34 & 34 & 34 & 34 \\
\hline
\end{tabular}

Tabla 5. Resultados del análisis de correlación. SPSS 
De esta tabla, se extrae las siguientes correlaciones lineales simples: ryx $1=-0,837$, $\mathrm{p}=0,0001$ (significativa); ryx $2=-0,890, \mathrm{p}=0,0001$ (significativa) y ryx $3=-0,939, \mathrm{p}=0,0001$ (significativa).

En estos resultados observamos que todas las correlaciones que se dan entre la Soportabilidad «Vacunos» y las demás variables han resultado significativas; sin embargo, la mayor correlación lineal se ubica entre la soportabilidad «Vacunos» y cantidad de equinos, resultando las otras dos correlaciones en forma similar pero son de menor intensidad. En otros términos, la baja del nivel de soportabilidad del área de pastizales de la quebrada de Quillcayhuanca es el resultado del incremento poblacional del ganado vacuno, ovino y caballar; esta última especie es la que indica un nivel mayor de correlación. El mapa agrostológico obtenido en la presente actualización y el obtenido en la investigación realizada por el Parque Nacional Huascarán (2000), muestra un incremento de la superficie con pastos en malas condiciones.

\section{DISCUSIÓN}

La investigación: «Evaluación del impacto ambiental del Proyecto especial: Carretera de Integración Interregional: Huaraz - Pumachaca», para el sector de Quillcayhuanca, vía que fue dividida en la quebrada en los siguientes sectores: Quillcayhuanca-Yanahuanca, Cayesh-Fondo Valle, Tullparraju-Fondo Valle Cayesh-Margen izquierdo, cuya área abarca, nos da información de vigor con un máximo de longitud de follaje del 57,70\% $48,63 \%$ 63,95\% y 62,93\%, respectivamente, y nos indica que la condición del pastizal se encuentra entre buena y regular (Flores y Malpartida, 1987). Comparándola con los datos obtenidos en la presente investigación se tiene evidencia significativa de que los pastos nativos vienen siendo sobrepastoreados cada vez más, de modo alarmante, debido al incremento de las especies de animales de pastoreo que se encuentran en la quebrada investigada. Los ensayos en Pastos Nativos, ejecutados por la UNA - La Molina en la región de Puno 1980, nos muestra que los niveles de soportabilidad de los pastos considerados Excelentes, comparados con los obtenidos en el ámbito de investigación, revelan una variación porcentual negativa del 75,00\% y 78,25\% con relación a lo obtenido en la investigación del año 2000 y la presente 2008, respectivamente, lo cual evidencia que nuestras praderas nativas se encuentran en un franco proceso de extinción de los pastos de mayor calidad agrostológica, debido fundamentalmente al sobrepastoreo. Como resultado de la obtención de las muestras de la parte florística, se pudo obtener siete muestras de suelo, suficientes para realizar un análisis, del cual se desprende que el nivel de $\mathrm{pH}$ de la quebrada de Quillcayhuanca se encuentra en un promedio de 5,11, nivel considerado aceptable para la agricultura. Como resultado de la evaluación de la composición florística de la quebrada de Quillcayhuanca, se estima una producción de materia verde (MV) por cada conglomerado en particular que varía de 480 a 4800 kg.MV/Ha. La utilización adecuada de materia verde MV es un paso muy importante, ya que a través de ella se puede estimar, en forma rápida, la condición 
del pastizal. Las especies vegetales que son apetecibles y tienen la mayor bondad para proporcionar buen forraje para la alimentación de los animales alpacunos y ovinos son la Stipa brachiphylla, Alchemilla pinnata (San Miguel y Serrahima, 2004), las que son calificadas como especies decrecientes. Existen otras especies que son medianamente apetecibles para los animales; los que son calificados como especies acrecentantes y las otras calificadas de indeseables, las cuales son consideradas las especies vegetales dominantes como Baccharis sp, Mublenbergia peruviana, Werneria nubigena y Lupinus sp. Estas especies son consideradas indeseables.

La demanda ganadera o carga animal en la zona de estudio está representada principalmente por ganado vacuno, ovino, y caballar, caracterización preponderante para el sobrepastoreo o «riesgo» de los pastos naturales (Leff, 1998). En el área de estudio no existe rotación de canchas de pastoreo para garantizar el buen manejo de los pastos que favorezcan la floración y la generación de semillas que garanticen su sostenibilidad en el ámbito de estudio. La falta de ciertas actividades culturales en la crianza extensiva es una de las causas del deterioro de la pradera natural (Salvador, 2002). La sobrecarga animal en el área, también provoca la migración de especies silvestres debido a la competencia para la alimentación con los animales domésticos.

\section{CONCLUSIONES}

Como resultado de la evaluación de la composición florística de la quebrada de Quillcayhuanca, se estima una producción de materia verde (MV) por cada conglomerado en particular; estos varían de 480 a 4800 kg.MV/Ha. La utilización adecuada de MV es un paso muy importante, ya que a través de ella se puede estimar, en forma rápida, la condición del pastizal.

Las especies vegetales que son apetecibles y que tienen la mayor bondad para proporcionar buen forraje de la alimentación de los animales alpacunos y ovinos son la Stipa brachiphylla, Alchemilla pinnata, las que son calificadas como especies decrecientes. Existen otras especies que son medianamente apetecibles para los animales; las que son calificadas como especies acrecentantes y las calificadas como indeseables, las cuales son consideradas especies vegetales dominantes como Baccharis sp, Mublenbergia peruviana, Werneria nubigena y Lupinus sp. Estas especies son consideradas indeseables.

La demanda ganadera o carga animal en la zona de estudio está representada principalmente por ganado vacuno, ovino y caballar, caracterización preponderante para el sobrepastoreo o «riesgo» de los pastos naturales. En el área de estudio no existe rotación de canchas de pastoreo para garantizar el buen manejo de los pastos, que favorezcan la floración y la generación de semillas que garanticen su sostenibilidad en el ámbito de estudio. La falta de ciertas actividades culturales en la crianza extensiva es una de las causas del deterioro de la pradera natural. La sobrecarga animal en el área también provoca la migración de especies silvestres debido a la competencia por la alimentación con los animales domésticos. 
Del total de superficie de pastos naturales 1725.77 has. (100\%) en la zona en estudio corresponden a una condición regular 670 has. (38,7\%), condición pobre 790 has. $(45,7 \%)$ y condición muy pobre 270 has. $(15,6 \%)$.

La condición de pastizal para ganado vacuno: pobre 600 has. y muy pobre 1130 has.

La condición de pastizal para ovinos: regular, 915 has., pobre 770 has. y muy pobre 45 has.

\section{REFERENCIAS BIBLIOGRÁFICAS}

Flores Arturo, Malpartida, Luis. 1987. «Manejo de Praderas Nativas, Pasturas Nativas y Pasturas en la Región Alto andina del Perú». Tomo I. Lima: Fondo del Libro del Banco Agrario.

Instituto Nacional de Recursos Naturales. 1995. «Mapa Ecológico del Perú» Guía Explicativa. Lima: Editorial INRENA.

Instituto Nacional de Recursos Naturales. 2000. «Estudio de Soportabilidad de pastos nativos de la quebrada Quillcayhuanca». Lima: Editorial INRENA.

Leff, Stuart. 1998. «Saber Ambiental. Sustentabilidad, racionalidad, complejidad, poder». Madrid: Siglo XXI Editores S.A.

Salvador, Martin. 2002. «Manual de Pastos Nativos del Parque Nacional Huascarán». Huaraz: Tarea Gráfica Educativa.

San Miguel, Serrahima. 2004. «Manual de Crianza de Animales». Lima: Lexus Editores.

Fecha de recepción: 6 de junio de 2016

Fecha de aceptación: 19 de diciembre de 2016

\section{Correspondencia}

Hernan Rolando Vega Mejia

vegamejia12@yahoo.es 